\title{
Tra tecniche poliorcetiche ed esegesi filologica: considerazioni intorno a Enea Tattico, Poliorketika 32, 3 (e 3, 6)
}

\author{
Francesco Fiorucci
}

\begin{abstract}
The investigation of technical aspects of Greek writings on siege matters may sometimes prove to be a very useful means to reconstruct their text. In paragraph 32 of his Poliorketika ("On Siegecraft") Aeneas Tacticus depicts the countermeasures to be taken against a direct assault on the city wall. The manuscript tradition, represented only by the Codex Laurentianus LV-4, preserves the word $\chi \varepsilon\{i n$ ('battlements'), but certain mechanical characteristics of the mentioned siege machines (like the battering ram) suggest that in this passage one must read $\tau \varepsilon i \chi \eta$ ('wall') instead, a correction already proposed by I. Casaubon in his editio princeps.
\end{abstract}

Keywords: Aeneas Tacticus; ancient Siege Warfare, philological note

È noto che i Poliorketika di Enea Tattico costituiscono un affresco straordinario sulla vita di una piccola comunità greca della metà del IV sec. a.C. sotto la minaccia di un assedio. Gran parte degli sforzi dell'autore è rivolta ad istruire i cittadini su come prepararsi a sostenere un blocco imposto dal nemico: tramite un'attenta regolamentazione delle attività quotidiane si mira a mantenere unita la comunità, nella convinzione che i pericoli maggiori possano giungere dall'interno. Soltanto nei paragrafi conclusivi di ciò che rimane dei Poliorketika (i quali, si ricordi, sono mutili del finale), e precisamente a partire dal capitolo 32, si inaugura "l'unica sezione unitaria sicuramente individuabile", come riconosciuto dalla critica, dedicata stavolta alle contromisure da adottare in previsione di attacchi portati direttamente contro le mura con l'ausilio di mezzi d'assalto, scale e operazioni di scavo' ${ }^{1}$.

$\mathrm{Ci}$ interessa ora focalizzare l'attenzione proprio sui consigli di Enea utili a fronteggiare il pericolo delle macchine d'assedio, e propriamente sul passo dove l'autore spiega come difendersi contro l'ariete e simili, capaci di abbattere le fortificazioni $(32,3)^{2}$ :

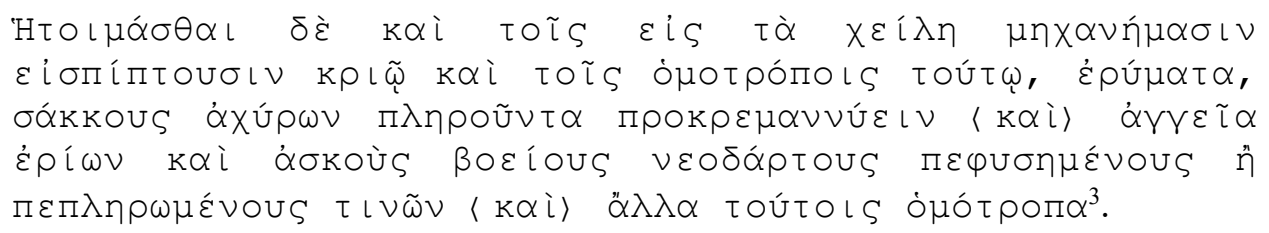

\footnotetext{
${ }^{1}$ La citazione è da BetTAlli $(1990,8)$. Da ricordare inoltre le fondamentali edizioni di HuNTERHANDFORD (1927) e DAIN-BON (1967); per una generale valutazione dell'opera vd. anche VELA TEJADA (1993); sulla divisione interna degli argomenti anche WHITEHEAD (1990, 17ss.); sui ricordati paragrafi finali rinvio a GARLAN (1974, 169-183) e FIORUCCI (2016), dove ho già proposto alcune note filologiche.

${ }^{2}$ Per ricevere un'idea dell'ariete nel mondo antico, gli episodi in cui entrò in azione e gli autori che ce lo descrivono, si rimanda a GARLAN (1974, 236-239) e al più divulgativo saggio di SÁEZ ABAD (2005, 89-93).

${ }^{3} \mathrm{Nel}$ presente studio si seguirà il testo stabilito da BETTALLI (1990), da cui sono tratte anche le relative traduzioni.
} 
Si devono anche preparare delle protezioni contro le macchine - arieti e simili che battono contro le merlature, sospendendo davanti ad esse dei sacchi pieni di paglia, canestri di lana e pelli fresche di bue, gonfiate o riempite in qualche modo, o altre cose ancora dello stesso genere.

Quasi tutte le edizioni accolgono il $\chi \varepsilon\{\hat{\imath} \eta$ della tradizione manoscritta, accettandone pacificamente il senso di 'parapetto' (Bettalli traduce "merlature", Hunter-Handford e Whitehead "battlements", Bon "créneaux"), cioè propriamente il bordo superiore del muro, che il termine verrebbe ad assumere ${ }^{4}$. Con questa indagine mi propongo invece di supportare la scelta del Casaubon, che nella sua editio princeps menda tramite $\tau \varepsilon i \chi \eta^{5}$.

Nonostante l'apparente ovvietà della soluzione proposta dall'umanista francese (del resto scartata, forse troppo sbrigativamente, dalla maggior parte degli studiosi), il problema merita una più approfondita revisione, che tenga conto di questioni sia filologiche sia (e soprattutto) di natura tecnica, quest'ultime determinanti, come vedremo.

Innanzitutto va detto che $x \in\{$ i $\lambda \circ \varsigma$, designando propriamente il 'labbro' e solo conseguentemente il bordo di un oggetto, si accompagna di norma ad un sostantivo che ne specifica il senso ${ }^{6}$. Un tale costrutto dovrebbe rivelarsi un'esigenza ancora più stringente in un caso come il nostro, dove si riscontra già un uso metaforico del termine, quindi andrebbe forse valutata la possibilità che sia caduto il genitivo $\langle\tau \circ \tilde{U}$ $\tau \varepsilon\{\chi \bigcirc \cup \zeta\rangle$, circostanza non considerata però dagli editori e ventilata marginalmente soltanto da Behrendt ${ }^{7}$. Un'ulteriore difficoltà nell'accettare $\chi \varepsilon i \chi \eta$ risiede nel fatto

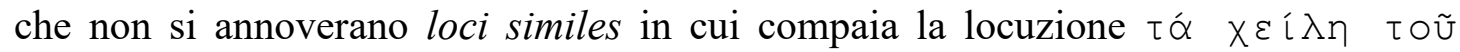
$\tau \varepsilon$ i XOU $S$, né l'uso del singolo vocabolo, anche in posizione isolata, col medesimo significato attribuito al passo dei Poliorketika. Come accennato, pochi si sono espressi a riguardo, ma le difficoltà non sono sfuggite ad editori come Hunter-Handford, i quali, pur lamentano l'assenza di paralleli, concludono che $x \varepsilon$ i $\lambda \eta$ "may very well be right" $"$. Va però notato che nei passi addotti da Hunter-Handford a difesa della lettura dei codici (Il. XII 52; Hdt. I 179, 2; Thuc. III 23, 2) l'oggetto di cui $\chi \varepsilon$ í $\bigcirc \circ \mathrm{S}$ costituisce il bordo viene regolarmente menzionato, trattandosi tra l'altro sempre del fossato ( $\tau \alpha \varphi \rho \circ \varsigma$ ), per cui siamo di fronte ad una iunctura ben attestata ${ }^{9}$.

\footnotetext{
${ }^{4}$ Anche GARLAN $(1974,171)$ sembra appoggiare l'interpretazione corrente, intendendo la sezione in esame nel modo seguente: "lèvres du mur, c'est-à-dire au parapet crénelé"; ma vd. anche ibid., p. 199. Più generica invece la resa "fortifications" di BATTISTINI-CHARVET-OZNAM $(1994,77)$. Il testo viene così riprodotto da tutti gli editori a partire da SCHÖNE (1911). L'unico testimone rilevante per l'opera di Enea è il Codex Laurentianus LV-4, tranne in alcuni punti dove questo risulta corrotto e possiamo avvalerci del contributo di copie successive: vd. DAIN (1935) e BETTALLI (1990, 49s).

${ }^{5}$ Vd. CASAUBON (1609 ad loc.), edizione posta in appendice a quella dell'opera storica di Polibio. La correzione ha trovato accoglienza, come accennato, nelle edizioni precedenti a quella di Schöne. Recentissimamente anche l'ultimo editore di Enea, cioè BRODERSEN (2017, 43 e 165), pur stampando il testo canonico, in entrambi i passi traduce rispettivamente "Mauerabschnitte" e "Außenmauern", evidentemente avvedendosi delle difficoltà insite in $x \in i \lambda \eta$.

${ }^{6}$ Vd. per esempio le valenze del termine suggerite in ThGL VIII coll. 1372-373 s.v. X $\varepsilon$ i $\lambda \circ$ S.

${ }^{7}$ Vd. BEHRENDT (1910, 71 n. 1).

${ }^{8}$ Vd. HUNTER-HANDFORD $(1927,112)$.

${ }^{9}$ Il testo tucidideo in III 23, 2 è stato inoltre posto sub iudice da $\operatorname{GOMME}(1956,285)$, il quale, ritenendo che la posizione assunta dai soldati sia in cima alle mura e non sull'orlo del fossato, propone di leggere

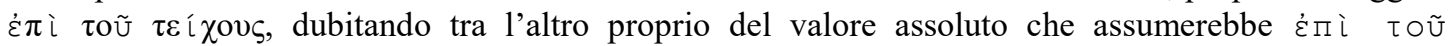

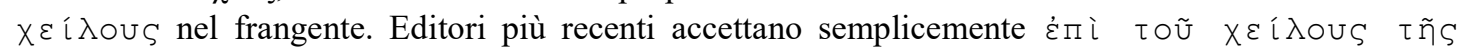

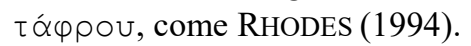


C'è da aggiungere che altrettanto poco convincente parrebbe anche il tentativo di additare in $\chi \varepsilon\{\hat{\imath} \lambda \eta$ un tecnicismo del registro poliorcetico, preso atto della suddetta mancanza di paralleli e considerando che, come testimonia tra gli altri Filone di Bisanzio (A, 14 ed. Garlan), ma il fenomeno si ripete costantemente anche al di fuori di questi autori tecnici, compare है $^{\prime} \alpha \lambda \xi\left\llcorner\varsigma\right.$ con il significato di 'parapetto' ${ }^{10}$.

Se dunque da un'analisi condotta con strumenti di tipo filologico-letterario la validità del testo tràdito rimane quantomeno dubbia e la sostituzione tramite $\tau \varepsilon i \chi\rceil \eta$ potrebbe apparire soltanto un seducente e comodo tentativo di normalizzazione, pare legittimo (direi anzi necessario) chiedersi se sia possibile aggiungere ulteriori considerazioni in base ad un approccio affatto diverso, muovendo cioè da rilievi di tipo tecnico e misurando l'enunciato di Enea secondo i parametri della prassi poliorcetica antica ${ }^{11}$.

Un criterio di giudizio basato su tali categorie sottintende ovviamente come presupposto l'assegnazione di un ampio credito all'accuratezza della nostra fonte (di cui del resto non abbiamo motivo di dubitare), ma offre al contempo puntelli piuttosto solidi su cui fondare le nostre speculazioni. Da un esame delle strategie difensive previste da Enea, infatti, scaturisce un quadro piuttosto nitido, in particolar modo per quanto concerne l'occorrenza in 32,3 , sulla quale conviene ora appuntare l'attenzione ${ }^{12}$. Tali strategie sono tutte votate a contrastare un assalto indirizzato senza dubbio verso la parte bassa o mediana delle mura e non contro i parapetti sovrastanti, il che naturalmente esclude la presenza di $\chi \varepsilon \hat{\imath} \lambda \eta$ inteso appunto con l'accezione sopra indicata ${ }^{13}$. La constatazione è convalidata in pratica da tutto il capitolo e in generale da quanto ci viene narrato nell'intera sezione finale dei Poliorketika. Enea, immediatamente dopo il passo citato all'inizio, prosegue infatti $(32,4-7)$ :

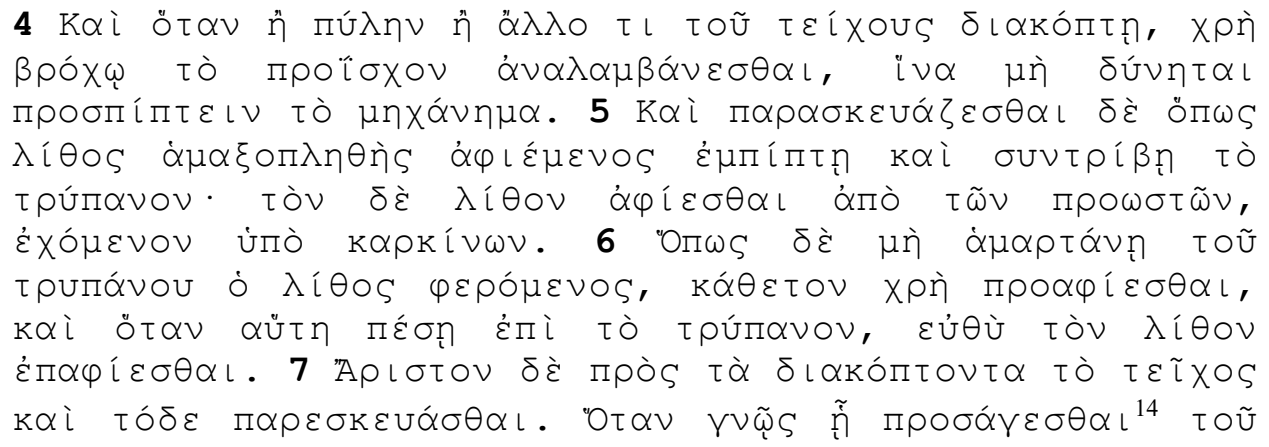

\footnotetext{
${ }^{10}$ Sulla lingua di Enea vd. HunTER-HANDFORD (1927, LII ss.) e VELA TEJADA (1991). In quest'ultimo saggio si tratta di $X \varepsilon\{\lambda \circ S$ e $\tau \varepsilon$ i XoS solo in relazione al fenomeno della contrazione (125s.). Sulla valenza semantica di $\varepsilon^{n} \Pi \alpha \lambda \xi\llcorner\varsigma$ si rimandi qui a WINTER (1971, 140s., con bibliografia) e GARLAN (1974, 344).

${ }^{11}$ La scelta di un efficace approccio metodologico non è ovviamente di secondaria importanza, tanto più nel caso di un testo come quello dei Poliorketika, su cui HunTER-HANDFORD (1927, 1xv) avvertono: “A 'correct' text of Aeneas is, no doubt, almost as impossible to arrive at as a 'correct' text of Homer; but it is hoped that the present attempt to emend him on historical principles may prove more successful than the reckless 'atticizing' to which he has been subjected".

${ }^{12}$ Per quanto riguarda $\times \varepsilon$ i $\lambda \eta$ in Enea 3, 6 si dirà meglio infra.

${ }^{13}$ Tra gli studiosi soltanto BETTALLI $(1990,318)$ si dimostra conscio della difficoltà, stigmatizzandola come segue: "Se la lezione $x \in \hat{\varepsilon} \backslash \lambda$ è giusta, dobbiamo dedurne che macchine del tipo degli arieti battevano anche contro le parti superiori delle mura".

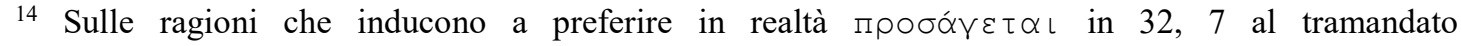

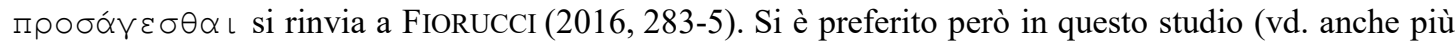
sotto) servirsi del testo di Enea come generale, sebbene molto perspicua, fonte di informazioni sulle pratiche poliorcetiche dei suoi tempi (già di per sé sufficientemente chiarificatrici ai nostri fini),
} 


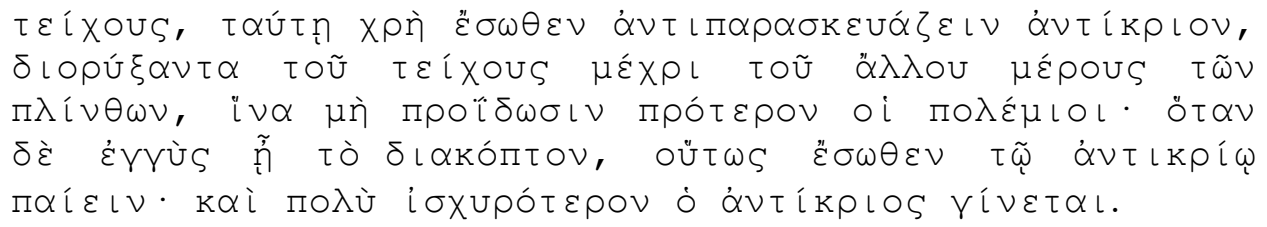

4 E quando viene aperta una breccia in una porta o in un altro punto delle mura, è necessario sollevare con una corda la testa dell'ariete, in modo che la macchina non sia più in grado di battere. 5 Bisogna anche tenersi pronti perché una pietra tanto grande da riempire un carro, lasciata libera, piombi giù e spezzi la punta dell'ariete: la pietra, tenuta ferma da ganci, deve essere fatta cadere dall'alto di travi sporgenti. 6 Perché la pietra, una volta lanciata, non manchi di colpire la testa dell'ariete, è necessario far scendere prima un filo a piombo; una volta che quest'ultimo sia caduto sulla testa, si lascerà subito andare la pietra. $7 \mathrm{Ma}$ la precauzione migliore da prendere contro le macchine che cercano di abbattere le mura è la seguente. Una volta localizzato il punto delle mura che sta per essere attaccato, bisogna preparare nello stesso punto, ma all'interno, un contro-ariete, scavando il muro fino ad arrivare solamente alla faccia esterna dei mattoni, in modo che i nemici non si accorgano in precedenza di queste manovre. Quindi, quando la macchina è vicina, allora si deve mettere in azione il contro-ariete all'interno: esso dovrà essere di gran lunga il più potente dei due.

I punti essenziali delle operazioni descritte si lasciano riassumere nel modo seguente:

1) il momento opportuno per sollevare l'ariete e neutralizzarne l'effetto è fissato quando questo sta per fare breccia nelle mura o in una porta $(32,4)$. Tale precisazione dimostra che la macchina batteva appunto contro il corpo del muro. Che senso avrebbe del resto colpire la parte alta delle porte? Ulteriori conferme giungono anche dall'analisi del lessico qui impiegato: va sottolineato infatti come Enea si serva

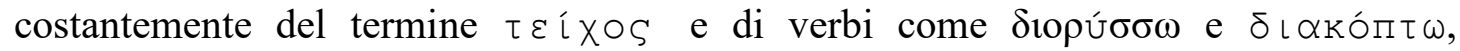
designanti l'azione di perforare e penetrare un ostacolo ${ }^{15}$;

2) per quanto riguarda nello specifico le contromisure per ostacolare il

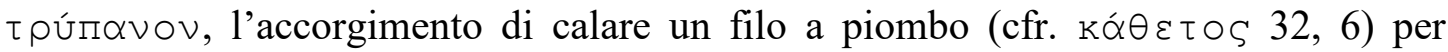
ridurre il rischio di mancare il colpo è prova del fatto che la distanza tra il punto di rilascio del masso (corrispondente all'incirca al bordo superiore del muro) ed il bersaglio doveva essere sufficientemente ampia da esigere una certa accortezza, considerando ovviamente anche la circostanza particolarmente caotica di un assedio: da ciò dobbiamo dedurre che la macchina assediante batteva non lontana dai piedi del muro $^{16}$;

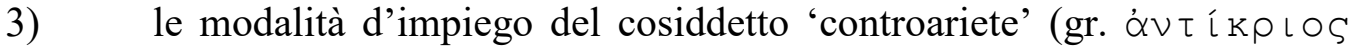
$32,7)$ evidenziano come il corrispettivo esterno non colpisse i parapetti: si parla

prescindendo dalle relative questioni filologiche, anche per non turbare il rapporto tra testo e annessa traduzione. La correzione пробó $₹ \varepsilon \tau \alpha \iota$ da me proposta è stata accolta nella recentissima edizione di BRODERSEN (2017).

${ }^{15}$ Un parallelo istruttivo per comprendere il senso di $\delta\llcorner$ ८ $\sigma \sigma \sigma \omega$ nel contesto è offerto dall'occorrenza in 32,11 , detto della testuggine scavatrice, che operava indiscutibilmente alla base delle fortificazioni (vd. anche infra).

${ }^{16}$ Si può anche essere d'accordo con HUNTER-HANDFORD (1927, 221), che reputano il suggerimento "a characteristic piece of ingenuity, though it seems rather superfluous", ma ciò che interessa verificare è la percezione del fenomeno testimoniata da Enea Tattico. 
infatti di come praticare un foro nelle mura e di come farlo a insaputa del nemico. Quest'ultima precisazione implica che l'operazione doveva avvenire sufficientemente al di sotto della linea tracciata dai parapetti, per sfuggire agli occhi indiscreti degli avversari ${ }^{17}$. Ancora, il dover raggiungere la parte esterna realizzata in mattoni chiarisce il tipo di composizione della cinta muraria cui Enea si riferisce ed implica che si tratti del corpo del muro ${ }^{18}$;

4) all'inizio del par. 7 l'autore specifica che certe contromisure sono da prendere contro quelle macchine che battono contro il muro. Il riferimento congiunto

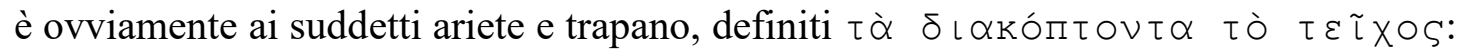
compare quindi esplicitamente il muro quale punto di impatto ${ }^{19}$.

Se quanto finora osservato non bastasse, possiamo verificare che il sistema difensivo imbastito da Enea non rappresenta un caso isolato, ma anzi contromisure analoghe sono attestate già in fasi storiche precedenti alla redazione dei Poliorketika, come dimostrano fonti di varia natura ${ }^{20}$. Tra queste spicca la ben nota testimonianza di Tucidide sull'assedio di Platea del 429, durante il quale le macchine degli assedianti furono messe fuori uso dai difensori tramite congegni che ricordano molto da vicino quelli descritti da Enea: si tratta sempre di pesi (travi nel caso di Platea) lasciati cadere oltre le mura (Thuc. II 76, 4):

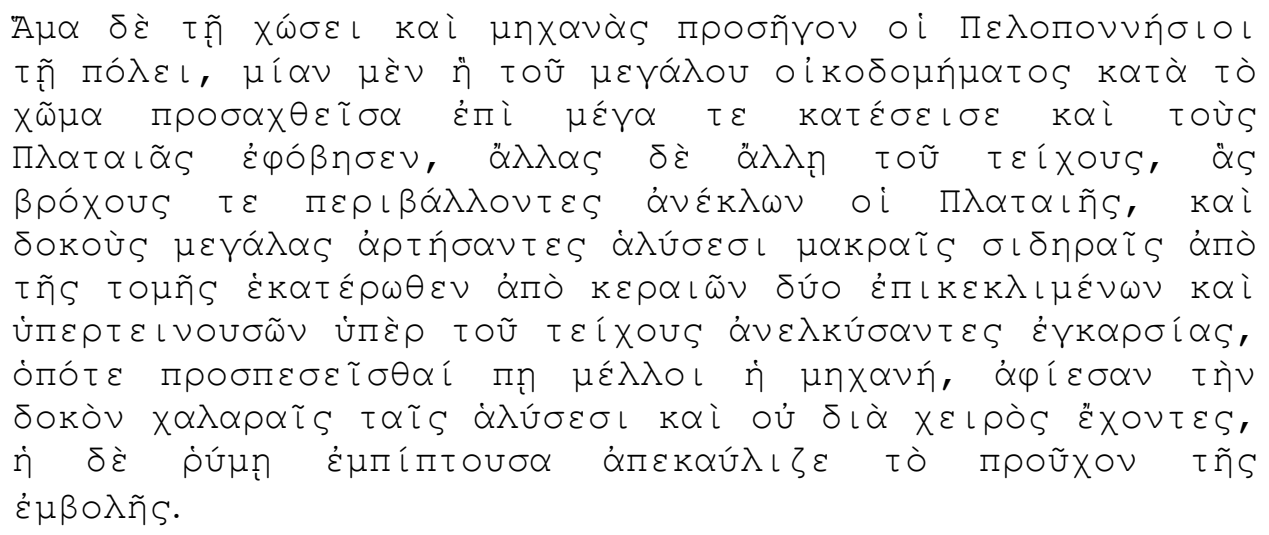

\footnotetext{
${ }^{17}$ Poco prima $(32,1)$ Enea aveva consigliato su come difendersi dagli attacchi portati dai nemici da posizioni elevate, da cui gli stessi avrebbero potuto spiare i preparativi degli assediati, qualora avvenuti sugli spalti. La necessità di tutelarsi dall'attività di sorveglianza del nemico era senz'altro un aspetto da non sottovalutare per la difesa. Si ricordi per esempio che Ateneo Meccanico istruisce su come dotare un tipo di testuggine riempitrice di una sovrastruttura che fungeva da osservatorio: righe 181-189 secondo la nuova numerazione della ed. $\operatorname{GATTO}(2010,221)$.

${ }^{18}$ In HunTER-HANDFORD $(1927,222)$ si spiega: "The words remind us of the ordinary construction of Greek walls, which consisted of two faces of masonry or brickwork, while the interior was filled up with rubble and loose material thrown in".

${ }^{19}$ Noto qui che hanno secondo me buone ragioni DAIN-BON $(1967,78 \mathrm{~s}$.) a distinguere nelle parole di Enea (32, 3-7) due diversi congegni, appunto l'ariete ed il trapano, come deducibile dal fatto che Enea

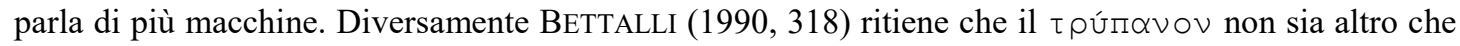
la punta dell'ariete.

${ }^{20}$ Vd. per esempio quanto mostrano dei rilievi assiri del IX sec. a C. in DUCREY 1986a (168-171, fig. 119 e 121). Ai nostri fini non è determinante il fatto che la fonte iconografica potrebbe rappresentare trapani e non arieti, parere fatto proprio da WHITEHEAD-BLYTH $(2004,80)$, perché ci interessa solo evidenziare la similitudine di certe contromisure rispetto all'opera di Enea (l'uso di catene o simili per sollevare il dispositivo avversario) e dimostrare che anche nel frangente gli aggressori prendevano di mira la parte bassa, o al più mediana, delle fortificazioni.
} 
Mentre costruivano il terrapieno, i Peloponnesiaci cominciarono a muovere contro la città anche le macchine d'assedio; una di queste, condotta su per il terrapieno, fece crollare buona parte della grande struttura, gettando i Plateesi nel terrore, mentre altre attaccarono altre sezioni delle mura. I Plateesi ne deviavano i colpi gettando attorno ad esse funi munite di cappi, oppure appendevano grosse travi, attaccate per le estremità a lunghe catene di ferro, a due pali inclinati che sporgevano dal muro, le sollevavano al di sopra del muro tenendole ad angolo retto rispetto alle macchine e, ogni volta che una di queste era sul punto di colpire, le lasciavano cadere allentando le catene e non trattenendole più: abbattendosi con violenza la trave troncava la parte sporgente dell'ariete ${ }^{21}$.

Le macchine trasportate dai Peloponnesiaci fin sotto le mura di Platea vengono concordemente identificate con gli arieti, come si evince del resto piuttosto palesemente dal resoconto ${ }^{22}$. I congegni sollevatori impiegati nella difesa della città dovevano sfruttare la più diretta dimensione a portata di mano, cioè l'altezza del muro. La posizione delle due antenne (che lo storico ateniese specifica fossero

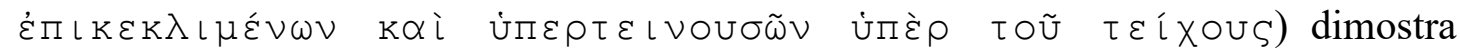
infatti che i pesi erano lasciati precipitare da una quota che doveva corrispondere all'incirca a quella delle stesse fortificazioni e ciò implica che il bersaglio doveva trovarsi, perché il colpo risultasse sufficientemente potente, a qualche distanza al di sotto di tale linea, laddove appunto battevano le macchine come l'ariete ${ }^{23}$.

Un confronto produttivo offre anche un passo di Polibio, che conferma come le modalità di impiego dell'ariete nelle operazioni ossidionali siano pressoché analoghe a quelle finora discusse. Stando al resoconto dello storico, Filippo V dovette pianificare un assedio in grande stile di fronte alle mura di Echino nel 210 a. C., ricorrendo all'ausilio di un complesso arsenale di macchine, tra cui lanciapietre e testuggini da riempimento, che avevano il compito di spianare la strada agli arieti. Per la nostra analisi ci interessa principalmente notare come l'autore distingua chiaramente la posizione di quest'ultimi, alla base della struttura portante, e quella delle misure necessarie per difenderli, dislocate sul terzo livello della stessa (Polyb. IX 41, 6):

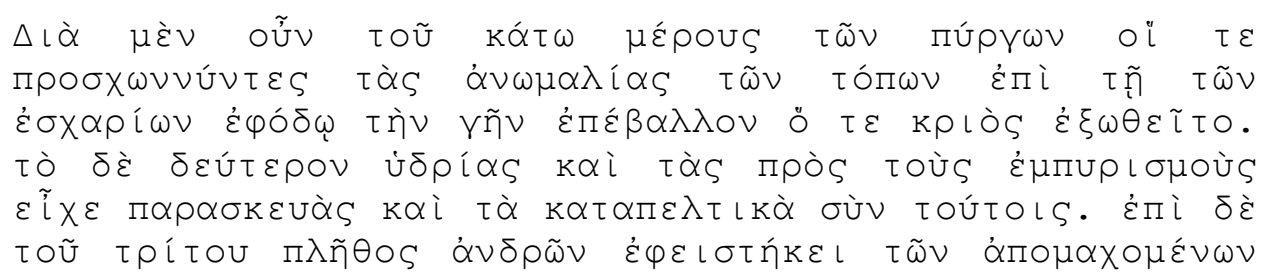

\footnotetext{
${ }^{21}$ Testo e traduzione da FANTASIA (2003, 171-173, vd. anche 534s. per le relative note di commento).

${ }^{22}$ Non è questa la sede appropriata per discutere nel dettaglio il funzionamento delle macchine qui coinvolte e per affrontare le varie questioni che il testo solleva, per cui basti rinviare a GOMME (1956, 209) e, per quanto concerne il rapporto del passo tucidideo coi Poliorketika, del resto universalmente riconosciuto, a BeTTALLi (1990, 317) e a GARLAN (1974, 137ss. e n. 7), con ulteriori riferimenti ad ulteriori testimonianze.

${ }^{23}$ Un altro celeberrimo episodio degno di menzione è quello dell'assedio di Siracusa ad opera di Marcello, durante il quale Archimede, per difendere la sua città, ideò tra gli altri congegni anche $i$ cosiddetti 'tolleni', famigerati tra le file degli assalitori, che sfruttavano i medesimi principi fisici delle macchine sollevatrici di Enea e di Tucidide: vd. LENDLE (1983, 120-7). Una testimonianza di un certo interesse si rivela anche Veg., mil. 4, 23, dove è chiaro che i difensori operavano direttamente dalle fortificazioni per sollevare in obliquo l'ariete: alii laqueis captos arietes, per multitudinem hominum de muro in obliquum trahunt, et cum ipsis testudinibus evertunt.
} 


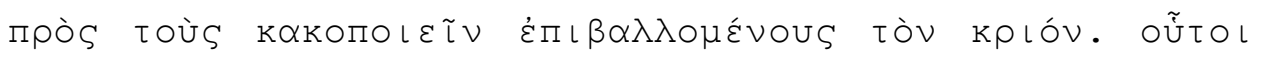

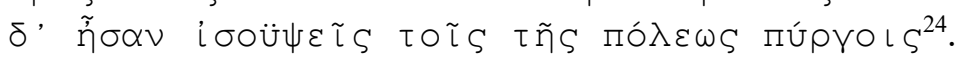

Per tutta la parte inferiore delle torri dunque gli addetti ad appianare le irregolarità del luogo gettavano terra per far avanzare le basi, e l'ariete veniva spinto. Il secondo piano aveva brocche d'acqua e tutto quanto predisposto contro gli incendi e insieme a queste cose anche le catapulte. Sul terzo livello si trovava invece un gran numero di uomini che combattevano da posizione elevata contro coloro che tentavano di danneggiare l'ariete. Questi erano alla stessa altezza delle torri della città.

L'esposizione è ancora una volta chiara: i soldati dell'ultima frase (unico riferimento possibile per il pronome ỡ்० ا) operavano all'altezza degli avversari disposti sulle mura, per poterne controbattere efficacemente le offensive, mentre l'ariete oscillava decisamente più in basso.

Tornando ora al testo oggetto della presente indagine, anche da una valutazione più generale delle difese contemplate da Enea nei paragrafi finali dei Poliorketika sembra giungere una conferma alle opinioni qui illustrate. Nel capitolo 33 l'autore avverte sull'efficacia del fuoco sia dal punto di vista della difesa sia da quello degli assalitori, cioè come arma per neutralizzare le macchine in avvicinamento, ma anche da cui bisogna tutelarsi grazie per esempio all'uso di schermi e materiali ignifughi, da applicare alle parti lignee delle fortificazioni (qualora presenti), che integravano ed evidentemente sormontavano la base in muratura $^{25}$. Si lasciano allora chiaramente distinguere due momenti nell'esposizione di Enea: dapprima (cap. 32) si contempla la difesa contro macchine di vario tipo in grado di abbattere le mura (dirette cioè contro il corpo delle fortificazioni o scavandone le fondamenta ${ }^{26}$ ), successivamente (cap. 33) vengono tematizzate le contromisure da prendere in caso di offensive condotte contro la parte alta delle fortificazioni, qualora queste fossero costruite in legno, dove gli arieti e i trapani perdevano con tutta evidenza la loro efficacia, ma il fuoco era da temere ${ }^{27}$.

L'esame della testimonianza di Enea secondo i criteri delle tecniche poliorcetiche antiche conduce ad ulteriori conclusioni. Se partiamo dall'ammissione che gli arieti e i trapani menzionati da Enea battessero contro le merlature, accettando quindi la lettura $x \varepsilon i \lambda \eta$, ne consegue giocoforza che questi fossero montati su una struttura di supporto sufficientemente alta e robusta da permettere loro di raggiungerle. Enea si limita in realtà a menzionare soltanto il corpo contundente, volendo focalizzare l'attenzione sulle misure difensive, da adottare appunto contro la trave, che costituiva l'elemento più appariscente e pericoloso dell'arma. Nonostante la mancanza di riferimenti a ulteriori componenti strutturali delle macchine, l'accorgimento difensivo che prevede il sollevamento della testa dell'ariete presuppone che quest'ultimo agisse sfruttando il principio dell'oscillazione, per cui doveva essere sospeso tramite funi o simili ad un qualche tipo di supporto mobile ${ }^{28}$.

\footnotetext{
${ }^{24}$ Sul passo vd. anche WALBANK $(1967,183 \mathrm{ss})$. La traduzione che segue è mia.

${ }^{25}$ Vd. BetTALLi (1990, 322) e WhitehEAD (1990, 197), con ulteriori rinvii.

${ }^{26}$ Sulle testuggini adatte a proteggere gli uomini addetti alle operazioni di scavo vd. infra.

27 Non va ovviamente dimenticato che la suddivisione interna dell'opera è moderna, sebbene pacificamente accettata dalla quasi totalità degli studiosi. Nel caso in esame la distinzione degli argomenti stabilita dall'autore è del tutto palese.

${ }^{28}$ Sul dettaglio tecnico della sospensione risulta istruttiva la breve storia dell'ariete tracciata da Ateneo Meccanico, righe 74-92 ed. Gatto, dove tra l'altro si narra brevemente dell'ingegno dimostrato da un certo Pefrasmeno, che avrebbe per primo intuito come sfruttare il principio dell'oscillazione per
} 
Allo stesso modo, le contromisure da opporre al trapano avvertono che questo non fosse probabilmente molto diverso da quello che conosciamo grazie ad Ateneo Meccanico (righe 127-139 ed. Gatto): era cioè alloggiato su un mezzo di trasporto o forse munito di una copertura ruotata ${ }^{29}$. Se dunque diviene facilmente ipotizzabile la presenza di una qualche forma di supporto per entrambe le macchine, permane il serio dubbio sul fatto che questo abbia loro consentito di raggiungere i parapetti ${ }^{30}$.

La questione della presenza o meno di macchine così complesse deve essere allora analizzata dall'unico punto di vista ammissibile scientificamente, cioè osservando lo sviluppo storico delle conoscenze tecniche in fatto di costruzioni di armi d'assalto nel mondo antico. Muovendo da tale assunto, possiamo isolare soltanto due passi, per quanto ci è dato ricostruire dalle notizie a nostra disposizione, in cui gli antichi ingegneri pare abbiano specificamente previsto l'uso di arieti contro la parte alta delle mura.

In primo luogo Ateneo Meccanico e Vitruvio ci hanno restituito la comune descrizione di una gigantesca testuggine arietaria scaturita dall'ingegno di un certo Egetore, la cui concreta realizzabilità e veridicità storica, va però subito precisato, è stata da più voci messa in discussione, tanto che potrebbe trattarsi di un modello che non superò mai la fase progettuale ${ }^{31}$.

L'altro caso compare in Apollodoro di Damasco (170, 3-6):

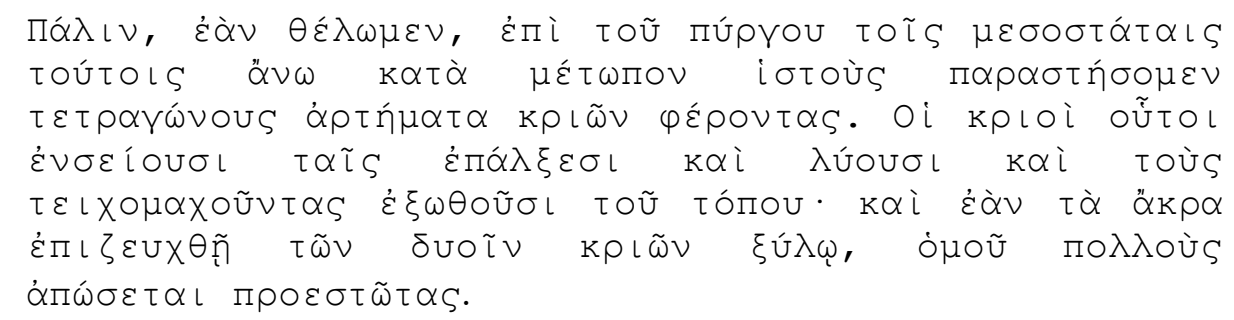

Inoltre, se vogliamo, metteremo sulla torre sopra i montanti del lato anteriore travetti quadrangolari che portano i pendagli dell'ariete. Questi arieti

\footnotetext{
incrementare la potenza dell'ariete. Per un commento sulle vicende ivi riportate si rinvia a WhiteHEAD-BLYTH (2004, 78-81) e GATTO (2010, 323-330, per un serrato confronto col corrispettivo passo vitruviano vd. anche $53 \mathrm{ss}$.).

${ }^{29}$ A proposito di questa macchina vd. LENDLE (1983, 128-132) e GATTO (2010, 424-428). Parzialmente divergente, ma senza che i punti di dissenso siano determinanti per le nostre riflessioni, la ricostruzione in WhitehEAD-BLYTh (2004, 103ss.), dove gli studiosi pensano ad un'arma capace di perforare le mura avversarie lavorando inclinata verso l'alto.

${ }^{30}$ Il compendio di Enea Tattico, a causa della sua natura manualistica, si prefigge di fornire un esauriente resoconto generale sulle contromisure considerate moderne ed efficaci ai tempi in cui è stato redatto, non consentendo di riconoscere, nella sezione finale qui analizzata, un qualsivoglia episodio storico da cui si possa valutare su basi più solide il tipo di costruzioni difensive interessate. Conosciamo tuttavia, grazie soprattutto all'archeologia, la situazione delle difese nelle città greche all'epoca di Enea. Da tali studi emerge che le mura potevano raggiungere altezze imponenti, pertanto le macchine in parola, qualora capaci di trasportare gli arieti fino ai parapetti, sarebbero dovute essere davvero enormi. Sulle fortificazioni greche rimando ai classici lavori di WINTER (1971) e LAWRENCE (1979); da integrare con vari contributi presenti in LERICHE-TRÉZINY (1986), tra cui segnalo GARLAN (1986); WiNTER (1986); DUCREY (1986b); MC NiCOLL (1986) 305-313.

${ }^{31}$ Soprattutto scettici WhiteHEAD-BLYTH (2004, 120-134 e 187-189); ma vd. anche LENDLE (1975, 48-86) e GATTO (2010, 395-420). Agli stessi saggi si rinvia anche per una valutazione della corrispettiva testimonianza vitruviana. Si noti incidentalmente che nelle considerazioni conclusive proprio sulla macchina di Egetore WHITEHEAD-BLYTH $(2004,188$ s.) sottolineano come il gigantesco ariete possedesse come caratteristica innovativa quella di poter colpire la parte alta delle mura, più debole rispetto a quella inferiore, diversamente dagli arieti convenzionali, utili solo contro il corpo delle fortificazioni. La delucidazione conferma indirettamente quanto finora sostenuto.
} 
investono i parapetti e li rompono, respingendo così coloro che lì difendono le mura; e se si collegano le teste di due arieti con un asse, con un colpo solo si scaraventeranno via molti difensori ${ }^{32}$.

Anche in questo caso è necessario cogliere fin da subito le sostanziali discrepanze tecniche che distanziano nettamente quanto qui descritto dalle macchine di Enea: secondo Apollodoro l'ariete è montato su una torre, quindi si trattava evidentemente di un mezzo di limitate dimensioni, dovendo essere manovrato dagli addetti disposti in cima a questa, e valido a smantellare solo il parapetto e colpirne i difensori, ma non in grado di fare breccia nelle mura, come invece doveva avvenire secondo le avvertenze di Enea. Inoltre ogni considerazione deve necessariamente tener conto delle recenti prospettive della critica, che hanno mosso pesanti dubbi sulla sua realizzabilità della macchina e sulla validità del passo ${ }^{33}$.

$\mathrm{Al}$ di là delle complesse (e per giunta non del tutto risolte) questioni che investono la natura stessa dei due dispositivi ora menzionati (la testuggine di Egetore e la torre di Apolldoro) ed il loro funzionamento, sulle quali non si pretende discutere in questa sede, bisogna innanzitutto tener conto di un fatto determinante, cioè che certe macchine si rivelano, concettualmente così come dal punto di vista del contesto storico che le ha (forse) generate, del tutto incongrue alle tecniche poliorcetiche esposte da Enea. I Poliorketika risalgono ad un periodo di parecchio anteriore rispetto all'età in cui queste macchine sarebbero entrate in azione, precedente all'avvento del regno macedone, che com'è noto segna un punto di svolta anche per la scienza degli assedi, soprattutto per opera di Alessandro Magno e degli ingegneri al suo seguito ${ }^{34}$.

A questo punto giova valutare con attenzione anche tutto l'impianto difensivo imbastito da Enea, che offre ulteriori puntelli a favore della tesi qui sostenuta. Anche nei Poliorketika, infatti, l'arsenale degli assalitori è dotato di imponenti strutture,

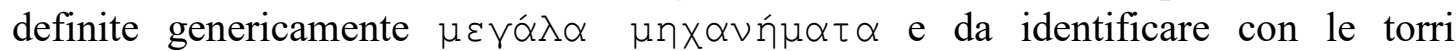
mobili ${ }^{35}$. A queste viene tuttavia affidato un compito tattico del tutto differente rispetto alle finalità per cui erano progettati i mezzi che figurano in Ateneo Meccanico o Apollodoro, cioè quello di consentire a uomini e mezzi leggeri come l'artiglieria di avvicinarsi alle mura, garantendo una piattaforma elevata da cui colpire con proiettili i difensori annidati sulle fortificazioni e raggiungere l'interno delle città.

Enea precisa a proposito $(32,8)$ :

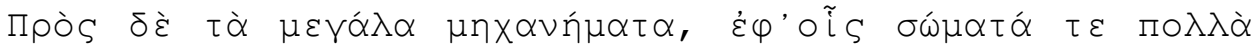

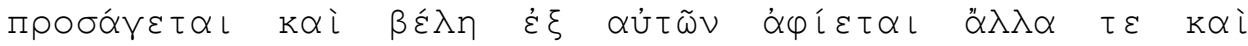

\footnotetext{
32 Testo e traduzione da COMMARE (1999, rispettivamente 36s. e 68).

${ }^{33}$ Vd. WhiteHEAD (2010, 115 ss.), che poggia i suoi ragionamenti su BLYTH (1992). Gli studiosi, forse con conclusioni francamente troppo nette, giudicano il passo interpolato, ponendo l'attenzione sul problema della stabilità della struttura, che verrebbe compromessa dall'oscillazione degli arieti.

34 Vd. KERN (1999, 197-226) e la recente sintesi di GATTO (2010, 18-23). Per essere ancora più rigorosi, va precisato che in realtà sulla datazione di Egetore non si ha certezza. Ad ogni modo, a prescindere dalle questioni sulla sua cronologia e nonostante il suo nome sia stato connesso a quello di Demetrio Poliorcete in pratica senza alcuna solida evidenza, come avvertono WHITEHEAD-BLYTH $(2004,120)$, non sussistono dubbi che la sofisticatezza della sua invenzione ha poco a che vedere con le testuggini menzionate poco dopo da Enea (vd. infra). Si deve comunque restare avvertiti del fatto che macchinari di grandi dimensioni in grado di abbattere le mura nemiche erano per esempio a disposizione degli eserciti assiri, come sopra brevemente ricordato, quindi in un periodo di molto anteriore a quello in cui Enea redige il suo manuale. La valutazione della nostra opera va però contestualizzata nella cornice dello sviluppo della poliorcetica in terra greca.

${ }^{35}$ Vd. per esempio BetTALLi $(1990,318)$ sulla distinzione tra questi congegni e i пúpYo l menzionati in 32,1 , nonché DAIN-BON $(1967,133)$.
} 


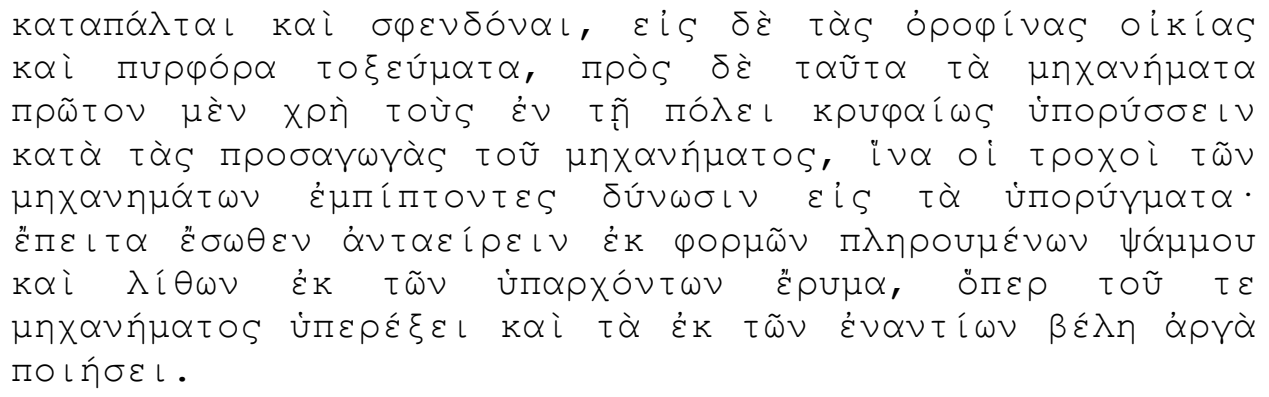

Contro i grandi macchinari, in grado di trasportare molti uomini e da cui strumenti da lancio quali catapulte e fionde fanno partire proiettili e anche frecce incendiarie indirizzate verso le case con tetti di canne; contro tali macchinari, dunque, bisogna innanzi tutto che gli abitanti della città scavino delle buche, in segreto, lungo le vie d'accesso delle macchine, in modo che le loro ruote ci caschino dentro e sprofondino. In secondo luogo, all'interno delle mura, si deve costruire un muro di protezione con ceste riempite di sabbia, o con le pietre che si riescono a trovare, il quale supererà in altezza la macchina e renderà inefficaci i proiettili nemici.

La concezione tattica che decide l'impiego delle torri emerge dai sistemi difensivi consigliati, consistenti nell'ostacolarne l'avvicinamento scavando segretamente dei fossati, e nel fornire le strutture sensibili al fuoco di una copertura contro i missili incendiari. Da ciò possiamo dedurre come, da un punto di vista difensivo, la minaccia principale derivasse essenzialmente dalla loro altezza e dalla loro capacità di avvicinarsi alle fortificazioni, mentre non viene fatto cenno alla possibilità che queste avessero di abbattere le mura. Tali 'grandi macchinari' non erano pertanto concepiti per trasportare arieti, per cui sarebbe del tutto capzioso ritenere che quest'ultimi potessero addirittura battere la sommità delle mura ${ }^{36}$.

Analoghe valutazioni sono estendibili anche alla macchina che, secondo quanto insegna la poliorcetica antica, era più appropriata all'alloggio dell'ariete, cioè la già ricordata testuggine ${ }^{37}$. Un modello di $x \varepsilon \lambda \omega ́ \nu \eta$ compare in effetti nell'arsenale bellico dei Poliorketika (32, 11-12):

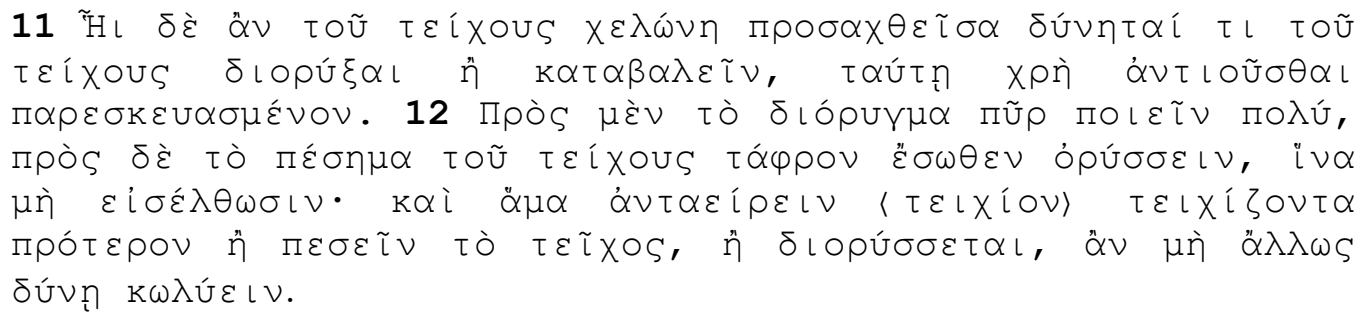

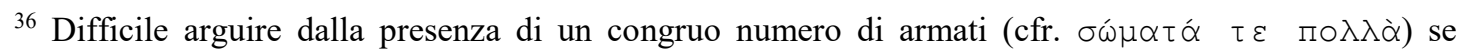
quest'ultimi siano semplicemente da identificare con gli addetti all'artiglieria appena menzionata o se l'autore abbia voluto piuttosto sottintendere la possibilità offerta da queste grandi strutture di ammassare quantità sufficienti di uomini in grado di assaltare le mura da posizione elevata. In tal caso sarebbe necessario l'ausilio di piattaforme appositamente predisposte, (chiamate comunemente $\dot{\varepsilon} \Pi \iota \beta \alpha ́ \theta \rho \alpha \iota$ o con simili designazioni e note da varie fonti, come Ateneo Meccanico e altri: vd. GATTO $(2010,355 \mathrm{~s}$.), di cui tuttavia Enea non fa parola. Comunque si desideri interpretare questo particolare, ci interessa aver verificato l'assenza di arieti in concomitanza con queste torri.

${ }^{37}$ Della macchina di Egetore si è accennato sopra, ma vd. anche la testuggine di Diade, restituitaci ancora da Ateneo Meccanico, righe 114-126 ed. Gatto.
} 
11 Nel punto, poi, in cui una testuggine, accostata alle mura, è in grado di aprire una breccia o di abbatterne una parte, lì bisogna essere pronti ad opporsi. 12 Contro la breccia, bisogna accendere un grande fuoco; contro un crollo del muro, invece, bisogna scavare un fossato all'interno, in modo da impedire l'irruzione dei nemici. Nello stesso tempo, prima che il muro crolli o venga scavata una galleria sotto di esso, se non è possibile evitare queste cose con altri mezzi, bisogna costruire un muro in seconda linea.

Le parole dell'autore lasciano margine a contrastanti interpretazioni sulle capacità operative di questa testuggine: può infatti venire connessa all'uso degli arieti, come sottintende BETTALLI (1990, 317 e 321 ad loc.), o piuttosto trattarsi, come propendo a credere, di un piccolo riparo disegnato per ospitare un ristretto gruppo di combattenti e consentire uno scavo manuale, cioè con l'unico ausilio di piccoli utensili, al riparo dai proiettili avversari ${ }^{38}$. Comunque si voglia intendere l'uso tattico di queste macchine, ciò che più conta nell'ottica del presente studio è che le difese previste (il ricorso al fuoco, lo scavo di un fossato o l'erezione di un secondo muro) possono esclusivamente essere messe in atto ai piedi delle fortificazioni.

Dopo aver dimostrato tutte le forzature che comporta la lettura $x \in\{\hat{\imath} \lambda \eta$ in 32,2 , è bene ora riprendere e concludere anche il discorso sul testo di 3, 6, lasciato precedentemente in sospeso ${ }^{39}$. Il testo recita:

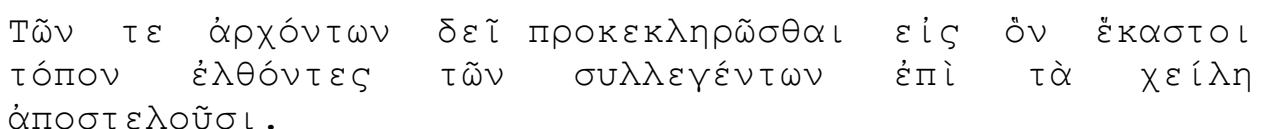

Bisogna sorteggiare in anticipo, inoltre, a qual punto di riunione deve recarsi ciascun comandante, perché possa ripartire sugli spalti delle mura gli uomini a disposizione ${ }^{40}$.

Dopo quanto abbiamo osservato sopra, dato che a $\chi \varepsilon \varepsilon\{\lambda \eta$ si attribuisce qui l'analogo significato che in 32,3 , ne consegue che le medesime perplessità sopra espresse devono estendersi anche a questo caso. Da un punto di vista paleografico, ciò implica che l'eventuale errore del manoscritto doveva comparire in entrambi i punti nella diretta linea di discendenza. Il fenomeno collima con quanto emerso dall'analisi della critica, visto che, come sostengono HUNTER-HANFORD (1927, xl), il codice M "even in the most impossible passages it shows no traces whatever of hesitation or confusion on the part of the writer: from which we must conclude that it is a very careful copy of another manuscript equally corrupt". Anche la natura dell'errore sembra compatibile con le caratteristiche riconosciute dagli studiosi come proprie del Laurentianus, tra cui spiccano per esempio confusioni tra parole graficamente affini e inversioni di lettere: fin troppo evidente è la somiglianza tra $x \in \dot{\imath} \lambda \eta$ e $\tau \varepsilon \dot{\imath} \chi \eta^{41}$.

\footnotetext{
38 Sarebbe cioè un modello di testuggine tatticamente e tecnicamente paragonabile alle piccole protezioni di cui parla Apollodoro di Damasco 143, 6ss. e su cui vd. WHITEHEAD (2010, 84SS.).

${ }^{39} \mathrm{Da}$ un punto di vista strettamente filologico, infatti, a difesa di $\chi \varepsilon$ i $\lambda \eta$ nel passo 32, 3 interviene (oltre ovviamente alla tradizione manoscritta) proprio la ricorrenza in 3, 6 con (supposto) analogo significato.

${ }^{40}$ Sul capitolo $3 \mathrm{vd}$. le note di commento di BetTALLi (1990, 220s.).

41 Una dissertazione ancora valida su certe peculiarità del manoscritto trovi in DAIN-BON (1967, XXXVIss.). Anche BEHRENDT (1910, 71 n. 1), sostenitore della stessa lettura qui difesa, commentando il passo 3, 6 individua in $x \in$ i $\lambda \eta$ una sicura corruttela innescata dai meccanismi sopra citati e apporta come prova un ulteriore caso in cui questo fenomeno si verifica: "Quare et 3, 6 et 32, $3 \times \varepsilon$ Ł $\lambda$ n ex
} 
Ancora, la presenza del termine $\chi \varepsilon\{i \lambda \eta$ in 3, 6 dimostra tutta la sua debolezza dal punto di vista del senso dell'enunciato in quanto la sua interpretazione come 'parapetto' dipende sostanzialmente da due fattori: in primo luogo dal confronto con la (congetturata) valenza in 32,3 (di cui abbiamo però appena esaminato tutte le debolezze) e in seconda istanza da quanto si legge poco sopra in 3, 1, dove il collaudato sistema del sorteggio viene questa volta applicato per provvedere all'assegnazione di una parte delle mura da difendere (come si vede sono ancora quest'ultime in primo piano!) alle rispettive $\varphi v \lambda \propto i$ in cui era ripartita la comunità cittadina:

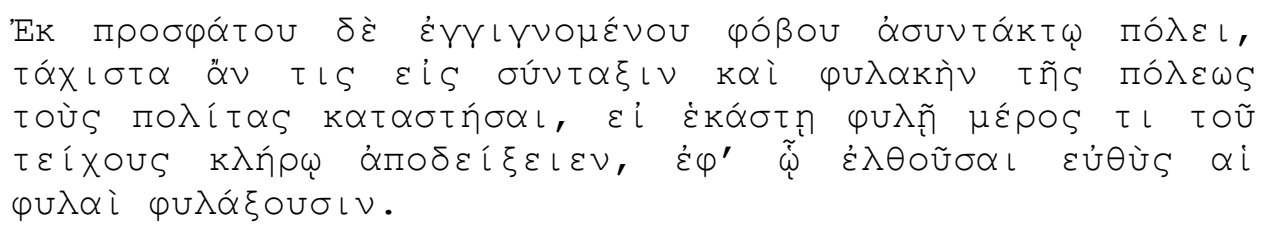

Se capita un improvviso allarme in una città militarmente impreparata, il sistema più rapido per organizzare i cittadini e difendere la città consiste nell'affidare a ciascuna tribù, per mezzo di un sorteggio, una parte delle mura, verso cui si dirigeranno immediatamente i suoi componenti per montare la guardia ${ }^{42}$.

Dato che il termine $\chi \varepsilon \varepsilon \hat{\imath} \lambda$ non possiede la pregnanza di un tecnicismo, come già constatato, se ulteriormente privato dei suddetti confronti la sua valenza in 3,6 risulterebbe oltremodo oscura ${ }^{43}$. Scrivendo $\tau \varepsilon \dot{\imath} \chi \eta$ anche in questo caso si otterrebbe invece un senso del tutto perspicuo e un ancor più diretto richiamo al precedente

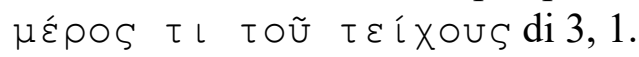

In conclusione, si può affermare che tutte le prove apportate rendono decisamente faticoso difendere il $\chi \varepsilon \varepsilon$ i $\lambda \eta$ dei codici in Enea Tattico 32, 3 e opportuna si rileva perciò la correzione del Casaubon nel più consono e comprensibile $\tau \varepsilon \dot{\imath} \chi \eta$. Dipendendo quindi la comprensione dell'occorrenza in 3, 6 sostanzialmente da quella in 32,3 , la medesima correzione va giocoforza applicata anche al caso precedente ${ }^{44}$.

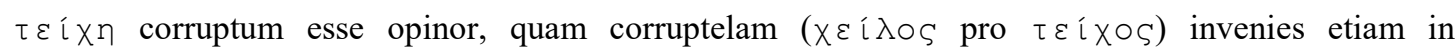
Thucydidis codice Heidelbergensi (E) III 23, 2, ubi sane quidem praecessit $x \varepsilon$ i $\lambda$ ous".

${ }^{42}$ Analoghe osservazioni valgono laddove in 3, 3 agli alleati viene assegnata una parte delle mura

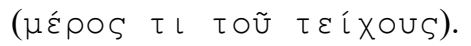

${ }^{43}$ Si noti come nella traduzione di 3, 6 l'editore abbia aggiunto "delle mura", conscio delle difficoltà relative al significato del solo $\chi \varepsilon$ i $\lambda \eta$, di cui si è discusso all'inizio.

${ }^{44}$ Un'ultima riflessione, non del tutto superflua, merita la forma del termine in esame, dovendo $x \varepsilon$ í $\lambda \eta$ essere necessariamente sostituito, per economizzare al massimo l'intervento, con il plurale $\tau \varepsilon$ í $\chi \eta$. Da un'analisi delle occorrenze del termine designante le 'mura' in Enea ci si accorge immediatamente che la forma preferita è senz'altro quella al singolare $\tau \varepsilon \tau \chi \chi \varsigma \zeta$, ma questa non è comunque di uso

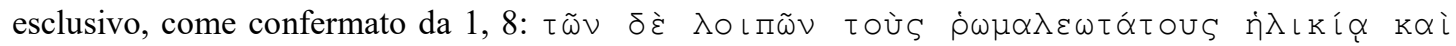

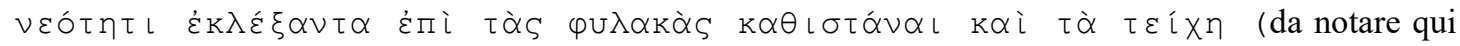

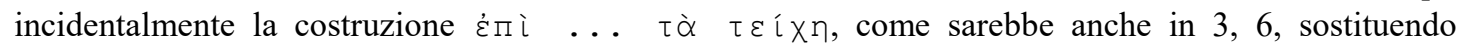
$\tau \varepsilon(\chi \eta)$. Ampliando la prospettiva dei riferimenti letterari, inoltre, ci si accorge che il plurale è di uso piuttosto comune in altri autori.
} 


\section{Bibliografia}

BATTISTINI-CHARVET-OZNAM 1994

O. Battistini-P. Charvet-A.-M. Oznam, La Guerre. Trois tacticiens grecs. Enée, Asclépiodote, Onasandre. Anthologie, Paris.

BEHRENDT 1910

C. Behrendt, De Aeneae Tactici Commentario poliorcetico quaestiones selectae, Königsberg.

BetTALLi 1990

M. Bettalli, Enea tattico. La difesa di una città assediata (Poliorketika), Pisa.

BLYTH 1992

P. H. Blyth, Apollodorus of Damascus and the Poliorcetica, «GRBS» 33, 1992, pp. 127-158.

BRODERSEN 2017

K. Brodersen, Aineias /Aeneas Tacticus. Poliorketika / Städteverteidigung, BerlinBoston.

CASAUBON 1609

I. Casaubon, Aeneae vetustissimi scriptoris Commentarius tacticus et obsidionalis, Parisiis.

COMMARE 1999

G. Commare, АПО $\Lambda \circ \triangle \Omega P O Y$ ПО IOPKHTIKA, in A. La Regina (ed.), L'arte dell'assedio di Apollodoro di Damasco, Milano.

DAIN 1935

A. Dain, Les Manuscrits d’Énée le Tacticien, «REG» XLVIII 1-32.

DAIN-BON 1967

A. Dain-A.-M. Bon, Énée le Tacticien. Poliorcétique, Paris.

DUCREY 1986a

P. Ducrey, Guerre et Guerriers dans la Grèce antique (1985), trad. ingl. Warfare in Ancient Greece, New York.

DUCREY 1986b

P. Ducrey, La fortification des cités grecques; rôle, fonction et efficacité, in P. Leriche-H. Tréziny (edd.), La fortification dans l'histoire du monde grec. Actes $d u$ Colloque International La fortification et sa place dans l'histoire politique, culturelle et sociale du monde grec. Valbonne, Dècembre 1982, Paris, 133-142.

FANTASIA 2003

U. Fantasia, Tucidide. La guerra del Peloponneso. Libro II, Pisa.

FIORUCCI 2016

F. Fiorucci, Note critiche al testo di Enea Tattico, in A. Setaioli (ed.), Apis Matina.

Studi in onore di Carlo Santini, Trieste, 283-294.

GARLAN 1974

Y. Garlan, Recherches de poliorcétique grecque, Paris. 


\section{GARLAN 1986}

Y. Garlan, Les fortifications grecques: bilan et perspectives de recherches, in $\mathrm{P}$. Leriche-H. Tréziny (edd.), La fortification dans l'histoire du monde grec. Actes $d u$ Colloque International La fortification et sa place dans l'histoire politique, culturelle et sociale du monde grec. Valbonne, Dècembre 1982, Paris, 15-21.

GATTO 2010

M. Gatto, Il חEPI MHXANHMAT $\Omega$ N di Ateneo Meccanico. Edizione critica, traduzione, commento e note. Roma.

GOMME 1956

A. W. Gomme, A historical Commentary on Thucydides, vol. II, Books II-III, Oxford.

HUNTER-HANDFORD 1927

L. W. Hunter-S. A. Handford, AINEIOY חOлIOpKHTIKA. Aeneas On Siegcraft, Oxford.

KERN 1999

P. B. Kern, Ancient Siege Warfare, Bloomington.

LAWRENCE 1979

A. W. Lawrence, Greek Aims in Fortification, Oxford.

LENDLE 1975

O. Lendle, Schildkröten. Antike Kriegsmaschinen in poliorketischen Texten, Wiesbaden.

\section{LENDLE 1983}

O. Lendle, Texte und Untersuchungen zum technischen Bereich der antiken Poliorketik, Wiesbaden.

\section{LERICHE-TRÉZINY 1986}

P. Leriche-H. Tréziny (edd.), La fortification dans l'histoire du monde grec. Actes $d u$ Colloque International La fortification et sa place dans l'histoire politique, culturelle et sociale du monde grec. Valbonne, Dècembre 1982, Paris.

\section{NICOLL 1986}

A. Mc Nicoll, Developments in techniques of siegecraft and fortifications in the Greek world ca. 400-100 B.C., in P. Leriche-H. Tréziny (edd.), La fortification dans l'histoire du monde grec. Actes du Colloque International La fortification et sa place dans l'histoire politique, culturelle et sociale du monde grec. Valbonne, Dècembre 1982, Paris, 305-313.

RHODES 1994

P. J. Rhodes, Thucydides. History III, Warminster.

SÁEZ ABAD 2005

R. Sáez Abad, Artillería y poliorcética en el mundo grecorromano, Madrid.

SCHÖNE 1911

R. Schöne, Aeneae Tactici de obsidione toleranda commentarius, Leipzig.

Vela TEJADA 1991

J. Vela Tejada, Estudio sobre la lengua de la Poliorcética de Eneas el Táctico, Zaragoza. 


\section{VeLA TEJADA 1993}

J. Vela Tejada, Tradición y originalidad en la obra de Eneas el Táctico: la génesis de la historiografía militar, «Minerva» VII 79-92.

WALBANK 1967

F. W. Walbank, A Historical Commentary on Polybius, vol. II, Oxford.

WHITEHEAD 1990

D. Whitehead, Aineias the Tactician. How to survive under Siege, London.

WHITEHEAD-BLYTH 2004

D. Whitehead-P.H. Blyth, Athenaeus Mechanicus, On Machines (Пєр 亡 $\mu \eta x \alpha \nu \eta \mu \alpha ́ \tau \omega \nu)$, Stuttgart.

WHITEHEAD 2010

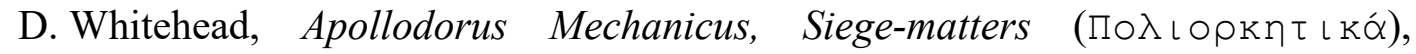
Stuttgart.

WINTER 1971

F. E. Winter, Greek Fortifications, Toronto-Buffalo.

WINTER 1986

F. E. Winter, A summary of recent work on Greek fortifications, in P. LericheH. Tréziny (edd.), La fortification dans l'histoire du monde grec. Actes $d u$ Colloque International La fortification et sa place dans l'histoire politique, culturelle et sociale du monde grec. Valbonne, Dècembre 1982, Paris, 23-29.

\section{Contatti:}

Dr. Francesco Fiorucci

Albert-Ludwigs-Universität Freiburg

Seminar für Griechische und Lateinische Philologie

Platz der Universität 3

D - 79085 Freiburg i. Br.

Email: francesco.fiorucci@altphil.uni-freiburg.de 